\title{
Septic Pulmonary Emboli With Feeding Vessel Sign
}

Tyler Kemnic, DO; Rohan Prasad, DO

From the Department of Internal Medicine at Michigan State University and Sparrow Hospital in East Lansing, Michigan.

Financial Disclosures: None reported. Support: None reported.

Address correspondence to: Tyler Kemnic, DO, Michigan

State University Clinical Center, 788 Service Rd, Room B-301, East Lansing,

MI, 48824-7013

Email: kemnicty@msu.edu

Submitted August 5, 2020; revision received August 17, 2020; accepted August 21, 2020.

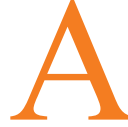
42-year-old woman with emphysema and a history of intravenous drug abuse and smoking presented to the emergency department for shortness of breath. She had a 2-week duration of dyspnea at rest, a left foot wound, and fevers. Initial vital signs were $103.8^{\circ} \mathrm{F}, 139 \mathrm{bpm}$, and $60 \mathrm{rpm}$. She required supplemental oxygen. Physical examination revealed diffuse bilateral wheezes and a left foot abscess. Blood cultures and polymerase chain reaction revealed methicillin-resistant Staphylococcus aureus (MRSA) bacteremia. Chest computed tomography angiography demonstrated diffuse bilateral pulmonary cavitation with the dominant lesion having a feeding vessel sign (image). The patient was diagnosed with MRSA endocarditis on echocardiogram with septic pulmonary emboli. Vancomycin was administered; however, the patient needed to be intubated. Lymphatic drainage was contraindicated in the patient because of the risk of systemic infection, bacteremia, and possible further dislodging emboli. ${ }^{1}$ Due to further decompensation, the family chose to pursue comfort care measures.

A septic pulmonary embolism is a blood vessel that is obstructed, usually by an infected thrombus. The pathogenesis consists of an embolic or ischemic event followed by an infection causing inflammation, which may form an abscess. ${ }^{2}$ Abscesses are most commonly caused by staphylococcal species, especially from infective endocarditis. ${ }^{3}$ On imaging, the "feeding vessel sign," also known as "fruits on the branch sign," is a combination of a distinct vessel leading directly to a nodular or mass. ${ }^{4}$ This finding can indicate one of the following: hematogenous origin near the small pulmonary vessels, a lung metastasis, or arteriovenous malformation. ${ }^{4,5}$ (doi:10.7556/jaoa.2020.130)

\section{References}

1. Franzini $D$, Curry LA, Pierce-Talsma S. Osteopathic lymphatic pump techniques. J Am Osteopath Assoc. 2018;118(7):e43-44 doi:10.7556.jaoa.2018.112

2. Stawicki SP, Firstenberg MS, Lyaker MR, et al. Septic embolism in the intensive care unit. Int J Crit IIIn Inj Sci. 2013;3(1):58-63. doi:10.4103/2229-5151.109423

3. Cook RJ, Ashton RW, Aughenbaugh GL, Ryu JH. Septic pulmonary embolism: presenting features and clinical course of 14 patients. Chest. 2005;128(1):162-166. doi:10.1378/ chest.128.1.162

4. Chiarenza A, Esposto Ultimo L, Falsaperla D, et al. Chest imaging using signs, symbols, and naturalistic images: a practical guide for radiologists and non-radiologists. Insights Imaging. 2019;10(1):114. doi:10.1186/s13244-019-0789-4

5. Dodd JD, Souza CA, Müller NL. High-resolution MDCT of pulmonary septic embolism: evaluation of the feeding vessel sign. Am J Roentgenol. 2006;187(3):623-9. doi:10.2214/ AJR.05.0681

(๑) 2020 American Osteopathic Association

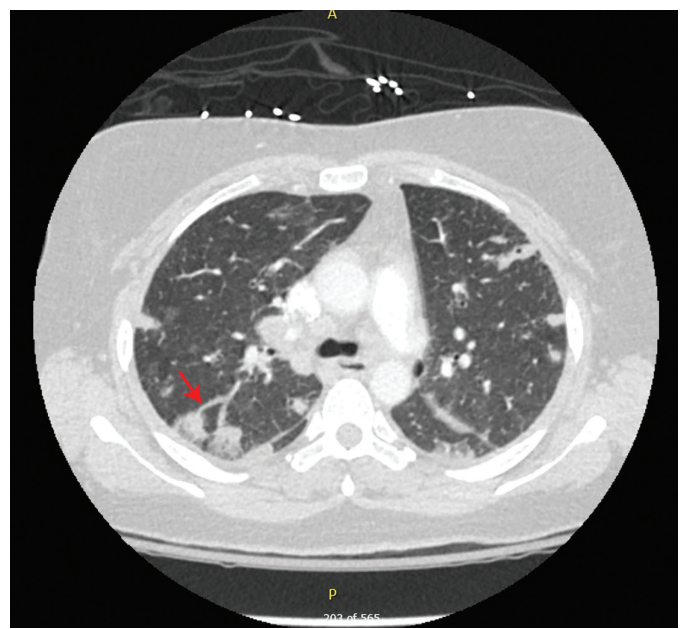

